\title{
Saccadic eye movements are deployed faster for salient facial stimuli, but are relatively indifferent to their emotional content
}

\author{
Abigail L M Webb ${ }^{1}$, Jordi M Asher ${ }^{1}$, Paul B Hibbard ${ }^{1}$ \\ 1 Department of Psychology, University of Essex, Colchester, Essex, UK \\ OThese authors contributed equally to this work. \\ * awebbc@essex.ac.uk
}

\begin{abstract}
The present study explores the threat bias for fearful facial expressions using saccadic latency as the response mode, with a particular focus on the role of low-level facial information, including spatial frequency, physical contrast, and apparent, perceived contrast. In a simple localisation task, participants were presented with spatially-filtered versions of neutral, fearful, angry and happy faces. Faces were either composed of naturally-occurring, expression-related differences in contrast, normalised for RMS contrast, or normalised for their apparent, perceived contrast. Together, findings show that saccadic responses are not biased toward fearful expressions compared to neutral, angry or happy counterparts, regardless of their spatial frequency content. Saccadic response times are, however, significantly influenced by the physical contrast of facial stimuli, and the extent to which these are preserved or normalised at the physical (RMS matched) and psychophysical (perceptually matched) level. We discuss the implications of findings for the threat bias literature, and the extent to which image processing can be expected to influence behavioural responses to socially-relevant facial stimuli.
\end{abstract}

\section{Introduction}

According to threat bias theory, visual information indicative of environmental threat receives prioritised access to visual processing [1 3]. Fearful faces are considered to play a unique sub-function of this threat-avoidance system, whose evolutionary role is to capture attention and facilitate its redirection towards the source of threat [1, 2, 4]. Biases for responding to fearful facial expressions have been explored using a great number of paradigms, and have produced mixed results. Studies of attentional cueing show both attentional capture and delayed disengagement by fearful faces [5, 6], attentional capture only [3,7,8, or an absence of these effects [8 11]; simple localisation tasks show that response times to detect fear expressions are faster compared to neutral faces [12,13], or no different 14, 15]; some studies show detection advantages for fear expressions masked by continuous flash suppression, backward-masking and sandwich-masking techniques 16,18 , while others do not [8,19]. It may be that paradigm-related differences can account for these mixed findings. Here, we focus on saccadic latency- a technique employed by a small number of threat bias studies, on the basis that it provides a naturalistic and ecologically-valid measure of the bias for fearful facial expressions. 
Saccadic latency refers to the first orienting step-like eye movement(s) deployed towards a visual stimulus, and how fast this occurs post stimulus onset 12 . A short time delay (measured in milliseconds) between the onset of a visual stimulus and the first successful saccade toward its location is taken as an indicator that the stimulus receives preferential allocation of attentional and/or processing resources. Saccades can be both explicitly directed towards that which is endogenously presented, or occur as implicit reflexive responses to transient, unexpected stimuli [3, 20]. These responses are therefore considered to be reliable measures of perceptual biases because they capture implicit and automatic behavioural responses in a way that mimics natural viewing, or in other words, they can capture eye movements towards faces as soon as they appear in the visual field, without the need to perform a calculated manual response. Despite this, compared to the wider literature on biases for detecting and responding to fear expressions there are relatively few studies that employ saccadic latency $6,11,13,21$, , and their findings are not always consistent. It can also be difficult to reconcile their findings because these studies use a variety of observer tasks, and so pursue biases for fear expressions in various contexts. For example, faster categorisiation of fear expressions [12] versus simple detection of stimuli presented after them, such as in an attentional cueing task 6, 11. In an emotion-directed localisation task observers were presented with two faces (neutral, fearful) on either side of the screen, and were directed to 'look towards' the location of the fearful face only [12. Bannerman et al. [12] showed faster saccadic initiation for fearful over neutral expressions, and that these effects were only observed when faces were shown for $20 \mathrm{~ms}$ (vs. $500 \mathrm{~ms}$ ). In an adaptation of this design, Bannerman et al. [6] incorporated an attentional cueing task, where fearful and neutral faces were presented as cues to target locations, and observers were required to respond only by looking towards the target as opposed to face cues. Their findings showed that saccadic responses were faster for targets following fearful face cues as opposed to neutral counterparts, and only when faces were available for $20 \mathrm{~ms}$ (vs. 100 ms). Using a similar attentional cueing design, however, Khalid et al. [11] also employed $20 \mathrm{~ms}$ presentations of neutral and fearful faces as cues to subsequently presented targets, but unlike findings from Bannerman et al. [6] fearful faces did not elicit faster saccadic reaction times compared to neutral faces, nor did they differ from saccadic responses to disgust faces [11. In a more straightforward free-viewing, or 'fast capture' task, observers were simply required to look toward the location of a face target shown for $20 \mathrm{~ms}$, and Bannerman et al [13] showed that saccadic responses were faster when the face was fearful versus neutral, but did not differ compared to response times for happy faces. Opposing findings, however, were observed in a different study by Seitz et al. [21] also employing a 'fast capture' task for $150 \mathrm{~ms}$ presentations of neutral, angry, fearful and happy faces. Initial saccades towards the eyes (as opposed to mouth) of faces were deployed faster for fearful compared to happy faces, though notably response times did not differ between fear and angry or neutral counterparts [21]. Together these findings emphasise the need for further replication, particularly for clarifying the effects of face presentation duration and task-type on saccadic responses to emotional facial stimuli.

Another factor to consider is the role of low-level image properties. Low-level, or image-based accounts of the bias for fearful expressions posit that it is the signal, or low-level image properties, of the expression that grant it prioritisation during visual processing, as opposed to its affective or social relevance 22 24]. The role of spatial frequency is common in these discussions, where a distinction is made between the lower frequency ranges that convey crude and coarse representations of a face, and the higher frequency information that depicts expression-related facial lines and nuanced information required for extracting fine facial detail. This focus reflects the potential role of specialised threat-processing mechanisms, for example in the amygdala [25], but 
also the idea that biases may reflect non-specialsed aspects of visual processing, for example the effects of the contrast sensitivity function [17]. Between various paradigms, findings show inconclusive frequency-dependent effects [8], where some studies show biased saccadic responses to low frequency fear expressions [13], while others demonstrate biases for high frequency fear expressions in detection tasks where faces are suppressed from conscious awareness [18,23], or in judgement tasks related to the perceived salience of faces [26]. Systematic investigations of saccadic responses to spatially-filtered facial expressions are rare [13], which is surprising given that this method is theoretically compatible with low-level accounts of the fear bias proposing that the prioritisation of fear expressions is likely to operate via crude and unconscious processing pathways 2, 13, functioning primarily to direct the observer towards the source of the threat. Furthermore, it is also important to explore how effects of image contrast could influence saccadic responses to fearful expressions, and facial stimuli generally. Normalising faces for RMS contrast removes naturally-occurring contrast differences between expressions, and for fearful faces in particular this process can artificially boost the degree of contrast required for a fearful expression to appear the same as other faces 26]. This disproportionate effect of contrast normalisation on already relatively low contrast fear expressions [26,27] is also influenced by spatial frequency filtering, such that high-frequency-filtered fear expressions, for example, require substantially less contrast than their low frequency counterparts to appear the same as a reference image 26 . Notably, these effects were most pronounced for stimuli normalised for their RMS rather than Michelson contrast 26]. These naturally occurring differences in the amplitude spectrum are important since one might expect that saccadic responses to faces would be significantly influenced by the physical and perceived salience of the stimulus, including its contrast and spatial frequency content.

In the present study, we measure saccadic responses to neutral, fearful, angry and happy expressions when filtered to contain low versus high spatial frequency content, and when presented in their natural, unfiltered, broadband state. Given that the effect of contrast normalisation, and the metric which is used to achieve this, affect expressions differently according to their spatial frequency content [26], we explored these effects for facial stimuli across 3 conditions, when raw and not matched for contrast, when normalised for Root Mean Squared (RMS) contrast, and when psychophysically matched for their apparent contrast. If it is the case that image salience, defined here as independent and interactive effects of contrast normalisation and spatial filtering, are an important feature for eliciting and guiding saccadic eye movements, then we should expect to see faster saccadic response times for physically salient facial stimuli, and the reduction of these effects when faces are made to be consistent at the apparent, perceived level. The present study is therefore an open exploration of effects related to stimulus creation and standardisation, and the extent to which they influence our initial eye movements towards the location of facial emotion.

\section{Materials and methods}

\section{Participants}

Twenty-three participants took part in the study (15 women), between 18 and 27 years of age. The University of Essex Ethics Committee approved the study and all participants gave written, informed consent. 


\section{Stimuli and apparatus}

Stimuli were presented using a Dell E2417H monitor, viewed from a distance of $80 \mathrm{~cm}$. The monitor screen was $48 \mathrm{~cm}$ wide and $27 \mathrm{~cm}$ tall, and had a resolution of 1920x1090 pixels and a refresh rate of $60 \mathrm{~Hz}$. Each pixel subtended 1.2 arc min, measured horizontally. The luminance response of the monitor was linearised using gamma-correction (Spyder 5 Elite, Datacolor). A chin-rest was used to maintain head position. Eye movements were recorded using an EyeLink 1000 eye-tracker (SR Research Ltd). Initial orienting saccades were recorded using the software's 'saccade start' function, defined as the point within a trial at which the saccade begins, measured in milliseconds. The sampling rate was $1000 \mathrm{~Hz}$. Eye movements were recorded from the right eye.

Stimuli were greyscale, front-view face images of 10 individuals ( 5 women) extracted from the Karolinska Directed Emotional Faces (KDEF) set 25], cropped to include internal features only. Each face portrayed 1 of 4 expressions(fear, anger, happiness or neutral). Spatially filtered versions of faces were created in MATLAB using a second-order Butterworth filter, with cut-off frequencies of $f<1_{c p d}$ for low frequency faces, and $f>6_{c p d}$ for high-frequency faces. These cut-offs match those used by $18,23,28$. Each face subtended $5.4^{\circ}$, such that the spatial amplitude spectrum of each face was either broadband, or was constrained to lower than 5 cycles per face-width (lowpass stimuli) or higher than 33 cycles per face-width (highpass stimuli). Three contrast versions of faces were created: (1) "raw" faces whose physical contrast was not adjusted in any way, such that any between-face differences were preserved, (2) Root Mean Squared (RMS) contrast-matched faces, and (3) psychophysically-matched faces, where each expression contained the degree of contrast necessary to appear to have the same contrast as a 10\% Michelson contrast standard. To create the final set of psychophysically-matched faces, we utilised data from a separate study in which participants (not associated with the present study) adjusted the physical contrast of facial stimuli -including the $10 \mathrm{KDEF}$ faces used here- in order to perceptually match them for their apparent, perceived contrast [26].

Differences in physical contrast between the three stimulus conditions (raw (unmatched), RMS-matched, and apparent-matched) are illustrated in Fig 1. It is important to note here that, due to the matching procedures used, raw stimuli are overall higher in physical RMS contrast compared to both RMS- and apparent-matched stimuli (both $p<.001$ ), but that the latter two conditions are not significantly different $(p=.17)$. We also know from previous findings that the raw (not normalised) stimuli set used in the present study contain naturally-occurring expression-related contrast differences 26, where unfiltered broadband fear expressions are naturally lower in RMS contrast compared to neutral and angry counterparts, lower in RMS contrast compared to happy faces only when filtered to contain low frequency information, and when high-frequency-filtered have lower RMS contrast compared to neutral, angry and happy expressions [26]. This naturally-occurring "deficit" in terms of contrast level for fearful faces is shown in Fig 1, and becomes visible as spatial frequency is increased. On the other hand, in terms of the apparent, perceived contrast for these same faces, we also know that broadband fearful expressions required significantly less RMS contrast than neutral and happy faces in order to appear perceptually the same, and less contrast compared to neutral and angry faces when high-frequency-filtered. When low-frequency-filtered, fearful faces do not significantly differ compared to others in terms of the amount of RMS contrast they require to appear the same 26]. This means that (i) the raw stimulus set used here contains the highest RMS contrast, (ii) they also contain naturally-occurring differences in RMS contrast across expressions, such that 
fear expressions are disproportionately lower in contrast, particularly as higher spatial frequencies, and (iii) when we normalise these same faces for their apparent, perceived contrast, the RMS contrast settings for fearful faces are lower than for other faces (particularly with increasing spatial frequency), because they require significantly less contrast than other faces to be perceptually matched. Lastly, by definition, faces normalised for RMS contrast were identical in terms of the contrast they contained, and we know that for these faces, fearful faces are likely to appear more salient than their counterparts, because the process of contrast normalisation has the effect of increasing the contrast required for these faces to appear matched [26].

Finally, faces were presented in one of two ways: in their natural, upright format, and in a manipulated format in which they were rotated $180^{\circ}$ and subjected to luminance polarity reversal. This method for creating control faces significantly disrupts facial emotion recognition while preserving low-level image properties including spatial frequency and contrast associated with the original, natural format of faces 22,29 . There are also mixed findings regarding the effect of luminance polarity reversal on the salience of faces, where some evidence implies that the increase in darker local regions enhances stimulus visibility 29]. Saccadic latency, as a measure of stimulus salience, is therefore a good candidate technique for further exploring the effect of luminance polarity reversal on face localisation. Stimuli were generated using MATLAB and the Psychophysics Toolbox extensions [?,30].

\section{Procedure}

All observers began the study with a 9-point calibration, according to the default EyeLink 1000 calibration program. Participants were instructed to press the space-bar when ready to begin a new trial. Each trial commenced with a single fixation cross appearing at the centre of the screen for a pseudorandom time interval between 250-1,000 ms. This fixation cross was immediately followed by a face target appearing to the left or right of centre, which remained on the screen for $200 \mathrm{~ms}$. The distance between the centre of each face and the centre of the screen was $7^{\circ}$. As a free-viewing task, observers were instructed simply to "look towards" the face image as soon as it appeared. Each trial was separated by a $200 \mathrm{~ms}$ gap, and before the onset of each trial, a default EyeLink 1000 drift correction, or re-calibration function, was used to ensure that each observer started each new trial from the same location e.g. by looking at the centre of the screen. There was a total of 720 trials (10 (actor) x 4 (expression) x 3 (frequency) x 3 (contrast) x 2 (orientation)). Trials were separated into 3 blocks according to spatial frequency (broadband, lowpass and highpass stimuli). The order of blocks was randomised between participants. The trial sequence can be seen in Fig 2 .

\section{Results}

Saccade responses were measured in milliseconds; shorter responses denote faster saccades towards faces. On trials where more than one saccade was made, only the first orienting saccade was extracted for data analysis. Data were analysed using a linear mixed effects model with the 'fitlme' function in MATLAB. For each model, random effects were defined over 'participant' and 'face image actor', due to possible variability related to individual differences, and the use of 10 randomly selected actors from the KDEF database. Categorical fixed effects for each model were 'expression' and 'face format', including the expression-by-format interaction, to measure the effects of 

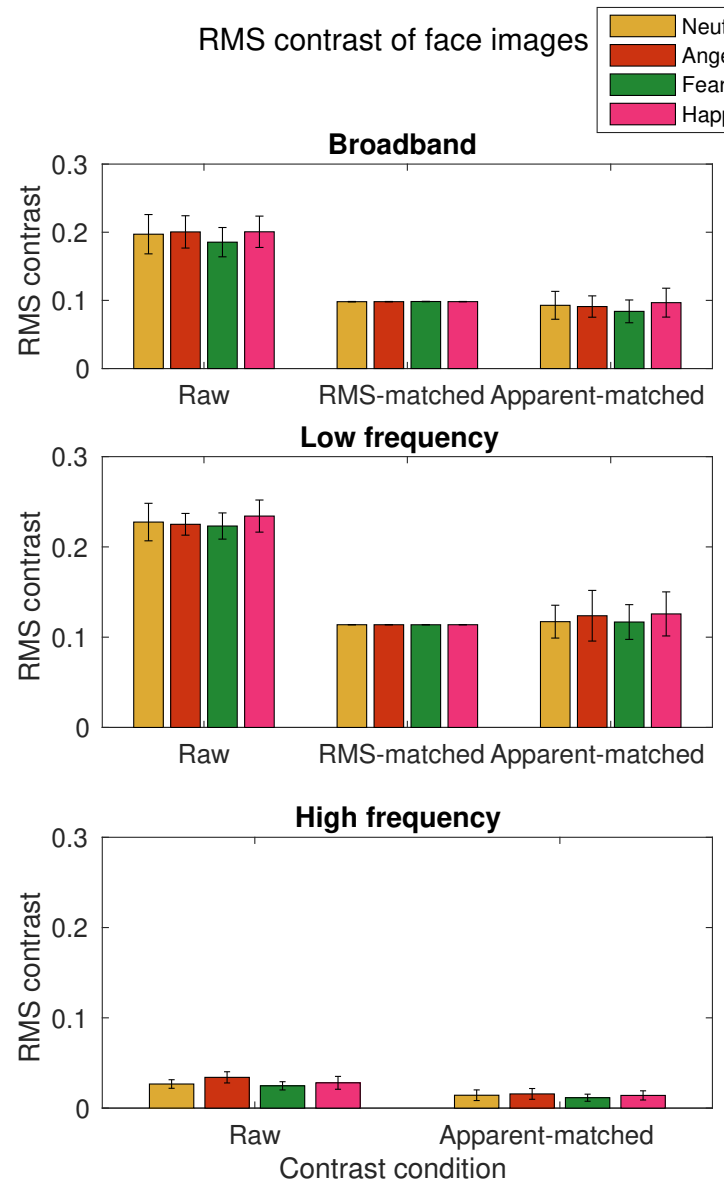

Fig 1. Expression-related differences in RMS contrast content across conditions for contrast, collapsed spatial frequency condition. Error bars represent standard deviations. 
Set up - 9 point calibration:

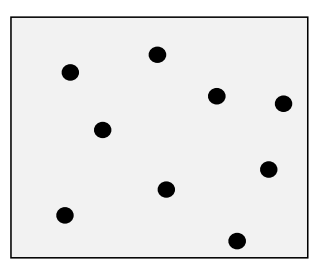

Trial sequence:

[drift correct]
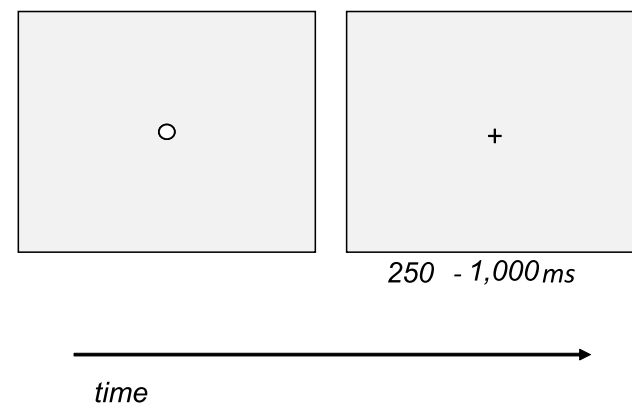

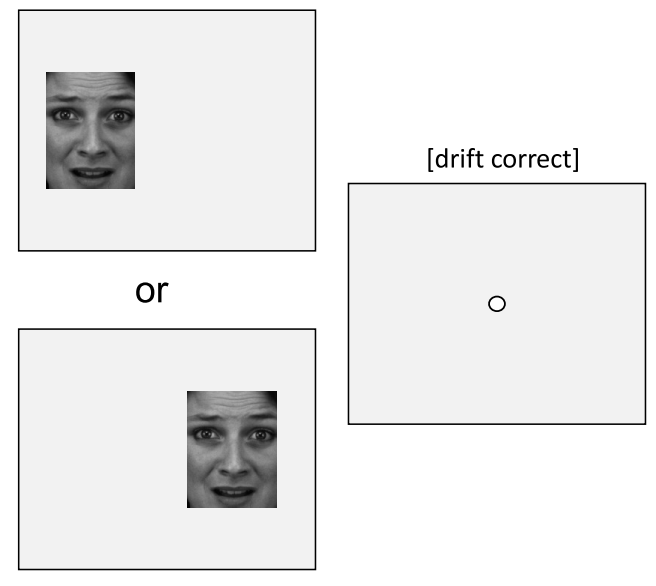

$200 \mathrm{~ms}$

Fig 2. Schematic representation of the task: each trial began with a central fixation point whose function was also to drift correct e.g. ensure that observers were looking at the centre of the screen. A fixation cross appeared for a pseudorandom time interval, followed by a facial expression to the left or right of the screen, appearing for $200 \mathrm{~ms}$. Participants were instructed to simply look towards the face as quickly and accurately as possible. 
face format manipulation on face detection between various emotions. Main effects of face format were assessed separately using the same model outlined above using 'face format' as the only fixed effect and no interactions. We include one model for broadband, low-, and high-frequency conditions at each of the 3 (raw, apparent matched, RMS matched) contrast conditions. Note that due to corrupted data the high-frequency-RMS-matched condition was excluded for all participants.

\section{Broadband facial stimuli}

Three linear mixed effects models are summarised in Table 1. Fig 3 shows saccadic responses for broadband facial expressions when they are (a) raw, and not normalised for contrast in any way, (b) normalised for RMS contrast, and (c) normalised for apparent, perceived contrast.

For raw (not normalised) broadband stimuli, saccadic responses for normally-presented (upright, retained luminance polarity) fearful faces did not differ compared to those for neutral, angry or happy faces. When the same faces were presented in manipulated format (inverted, reversed luminance polarity), saccadic response times for fearful expressions were significantly slower for fearful compared to neutral and angry faces, and also compared to normally-presented versions of fear. The overall main effect of face format, pooled across expression, was not significant, such

that saccadic response times did not differ between normal and manipulated versions of the faces.

For broadband faces normalised for RMS contrast, saccadic responses for normally-presented fearful expressions did not differ compared to those for neutral, angry, or happy faces. When the same faces were presented in manipulated format, saccadic responses for fearful expressions were significantly faster compared to manipulated neutral and angry faces. The overall main effect of face format was non-significant, such that saccadic response times did not differ for normal versus manipulated faces.

For broadband faces normalised for apparent, perceived contrast, saccadic response times did not differ between expressions, regardless of the format in which they were presented. There were no overall significant effect of face format.

\section{Low-frequency facial stimuli}

Three linear mixed effects models are summarised in Table 2, Fig 4 shows saccadic responses for low-frequency facial expressions when they are (a) raw, and not normalised for contrast in any way, (b) normalised for RMS contrast, and (c) normalised for apparent, perceived contrast.

For raw (not normalised) low frequency stimuli, saccadic responses for normally-presented fearful faces (upright, retained luminance polarity) did not differ compared to those for neutral, angry or happy faces. When the same faces were presented in manipulated format (inverted, reversed luminance polarity), no significant differences in saccadic response times were observed. The overall main effect of face format, pooled across expression, was also not significant, such that saccadic response times did not differ between low frequency normal and manipulated face versions.

For low frequency faces normalised for RMS contrast, saccadic responses for normally-presented fearful expressions did not differ compared to those for neutral, angry, or happy faces. Furthermore, no differences were observed when the same faces were presented in manipulated format, and the overall main effect of face format was non-significant, such that saccadic response times did not differ for normal versus manipulated faces. 

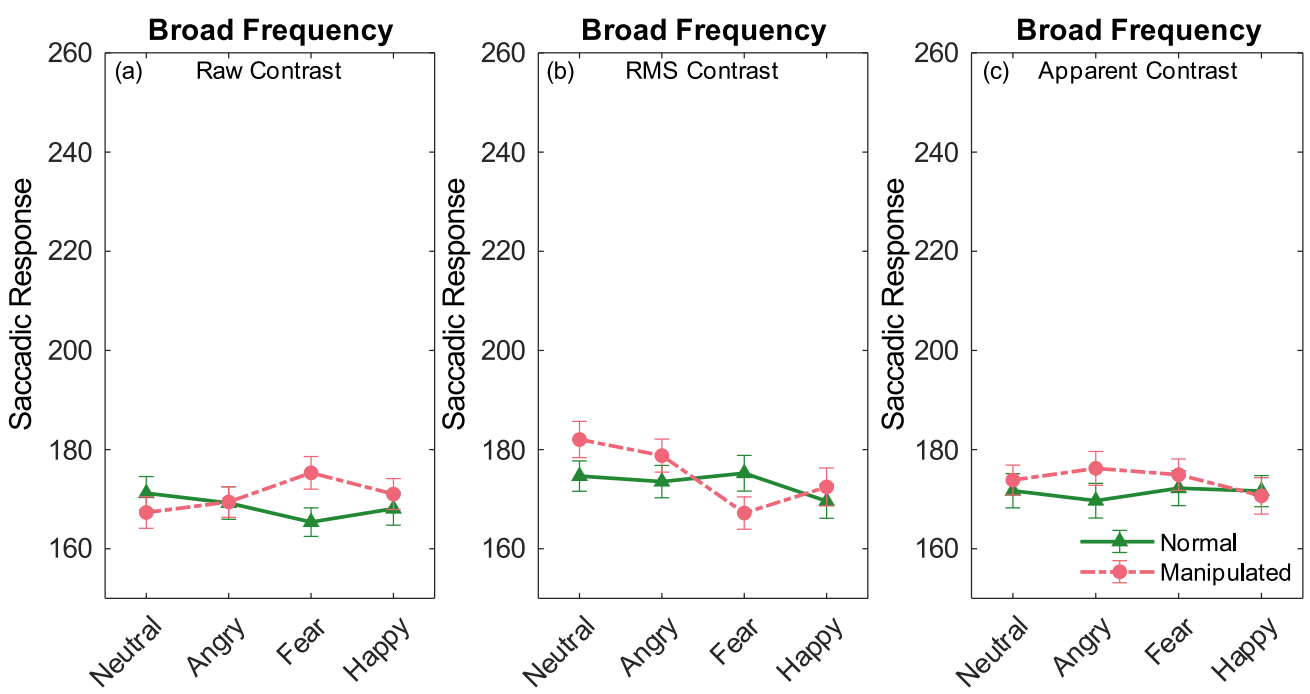

Fig 3. Saccadic responses (ms) for broadband-frequency facial stimuli in response to expressions; Neutral, Angry, Fear and Happy. Individual plots show responses to face-format; (a) raw and (c) normalised for RMS contrast. Green responses are for upright faces and orange are for manipulated faces (face format). Error bars are $1 \pm$ SEM.

For low frequency faces normalised for apparent, perceived contrast, saccadic responses for normally-presented fearful expressions did not differ compared to those for neutral, angry, or happy faces, and no significant differences were observed when the same faces were presented in manipulated format. The overall main effect of face format, however, was significant, where saccadic responses for normally-presented faces were overall faster compared to manipulated versions, when pooled across expression.

\section{High-frequency facial stimuli}

Two linear mixed effects models are summarised in Table 5 . Fig 5 shows saccadic responses for high-frequency facial expressions when they are (a) raw, and not normalised for contrast in any way, or (c) normalised psychophysically for apparent, perceived contrast. Note that data for high frequency RMS-normalised faces were corrupted due to programming error, and so are not presented here.

For raw (not normalised) high frequency faces, saccadic responses for normally-presented stimuli (upright, retained luminance polarity) did not differ compared to those for neutral, angry or happy faces. When the same faces were presented in manipulated format (inverted, reversed luminance polarity), no significant differences in saccadic response times were observed. The overall main effect of face format, pooled across expression, was significant, where saccadic response times were faster for normally-presented raw broadband faces than for manipulated counterparts.

For high frequency faces normalised for apparent, perceived contrast, saccadic responses for normally-presented fearful expressions did not differ compared to those for neutral, angry, or happy faces. Furthermore, no differences were observed when the same faces were presented in manipulated format, and the overall main effect of face format was non-significant, such that saccadic response times did not differ for normal versus manipulated high frequency faces. 

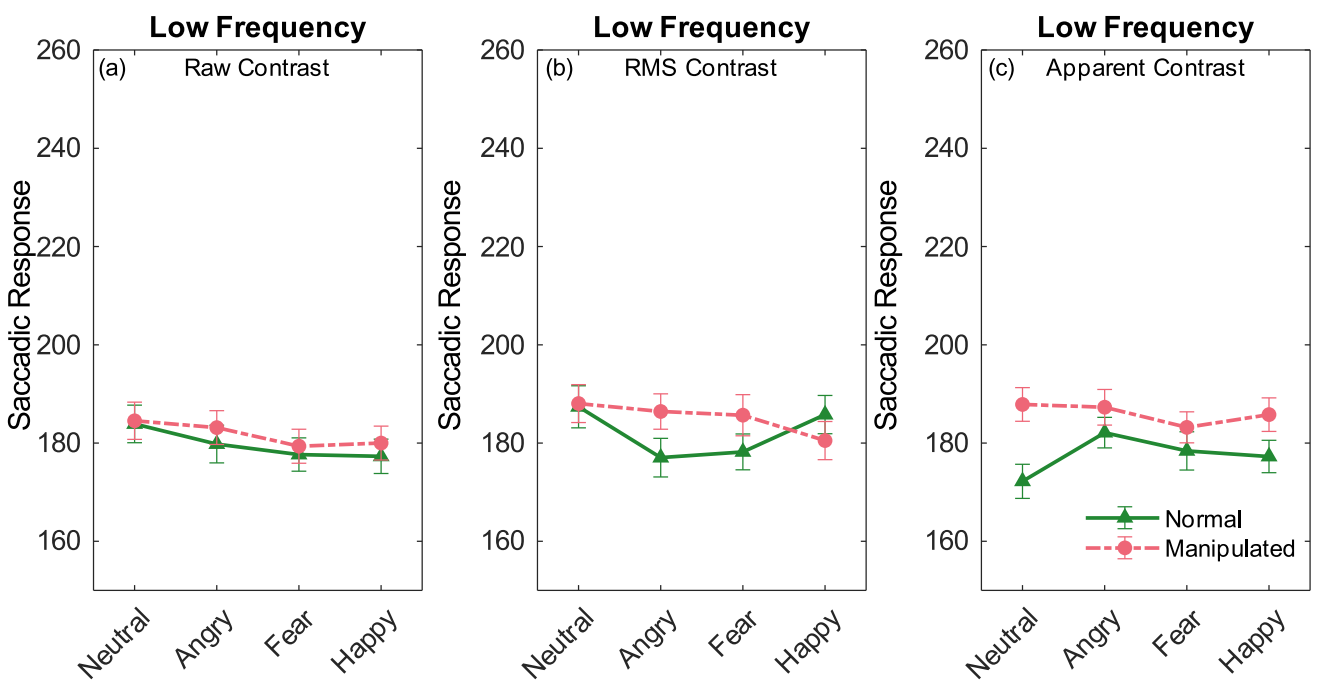

Fig 4. Saccadic responses (ms) for low-frequency facial stimuli in response to expressions; Neutral, Angry, Fear and Happy. Individual plots show responses to face-format; (a) raw and (c) normalised for RMS contrast. Green responses are for upright faces and orange are for manipulated faces (face format). Error bars are $1 \pm$ SEM.
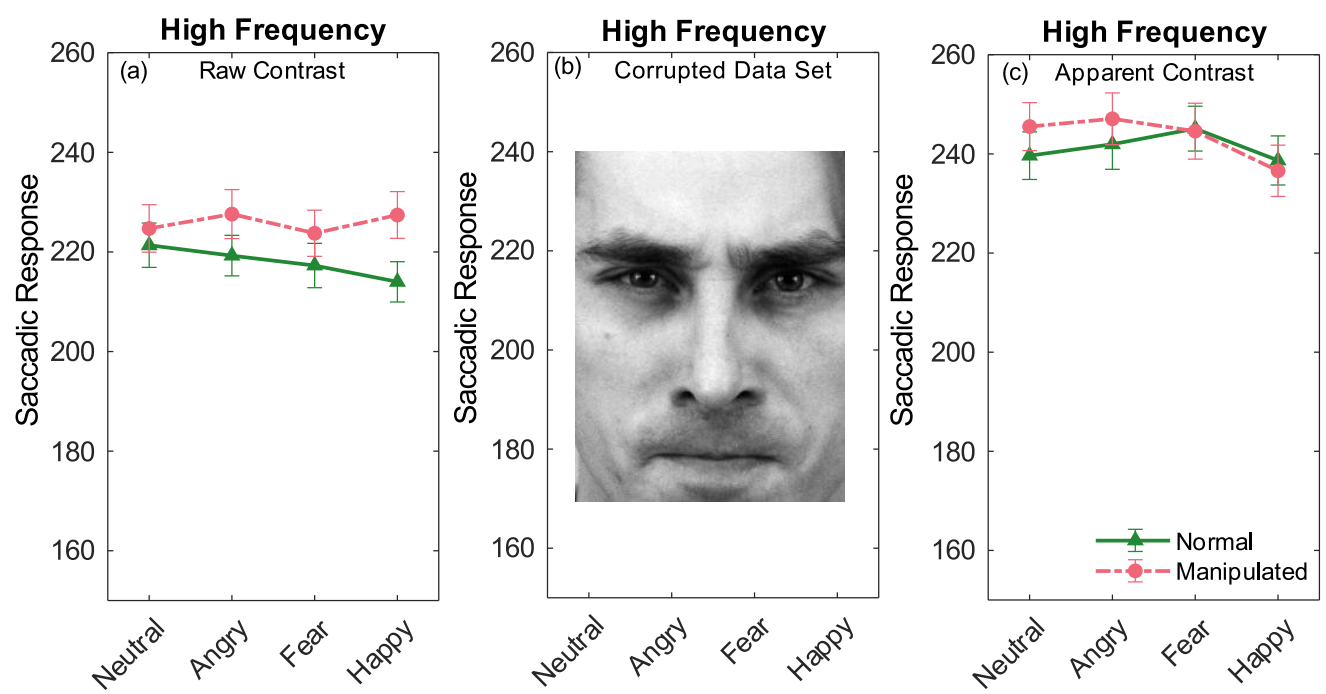

Fig 5. Saccadic responses (ms) for high-frequency facial stimuli in response to expressions; Neutral, Angry, Fear and Happy. Individual plots show responses to face-format; (a) raw and (c) normalised for RMS contrast. Green responses are for upright faces and orange are for manipulated faces (face format). Error bars are 1士 SEM. Note there is no plot for for normalised responses. 
Table 1. Linear mixed effects models (3) summarising saccadic responses for broadband facial stimuli, at each contrast condition.

\begin{tabular}{|c|c|c|c|c|c|c|}
\hline & Response estimate (ms) & SE & tStat & df & $p$ & CI (95\%) \\
\hline \multicolumn{7}{|c|}{ (a) Saccadic responses to raw (unmatched), broadband faces } \\
\hline \multicolumn{7}{|c|}{ Expression effect - Normal Faces } \\
\hline Intercept (Fear) & 165.39 & 5.16 & 32.01 & 1832 & $<.001$ & $155.26,175.52$ \\
\hline Fear-Neutral & $+5.82(171.21)$ & 4.08 & 1.42 & 1832 & 0.15 & $-2.18,13.83$ \\
\hline Fear-Anger & $+3.84(169.23)$ & 4.08 & 0.94 & 1832 & 0.35 & $-4.17,11.84$ \\
\hline Fear-Happy & $+2.7(168.09)$ & 4.08 & 0.66 & 1832 & 0.50 & $-5.31,10.71$ \\
\hline \multicolumn{7}{|c|}{ Expression effect - Manipulated Faces } \\
\hline Fear-manipulated & $+9.94(175.33)$ & 4.08 & 2.43 & 1832 & $0.01^{*}$ & $1.93,17.94$ \\
\hline Neutral-manipulated & $-13.83(161.50)$ & 5.77 & -2.39 & 1832 & $0.02^{*}$ & $-25.15,-2.50$ \\
\hline Anger-manipulated & $-9.72(165.61)$ & 5.77 & -1.68 & 1832 & $0.01^{*}$ & $-21.05,1.60$ \\
\hline Happy-manipulated & $-6.70(168.35)$ & 5.77 & -1.21 & 1832 & 0.23 & $-18.31,4.34$ \\
\hline Main Effect of Manipulation & $(168.48-170.79)$ & 2.05 & 1.13 & 1838 & 0.26 & $-1.71,6.32$ \\
\hline \multicolumn{7}{|c|}{ (b) Saccadic responses to RMS-matched, broadband faces } \\
\hline \multicolumn{7}{|c|}{ Expression effect - Normal Faces } \\
\hline Intercept (Fear) & 175.24 & 5.43 & 32.25 & 1832 & $<.001$ & $164.58,185.90$ \\
\hline Fear-Neutral & $-0.57(174.67)$ & 4.43 & -0.13 & 1832 & 0.90 & $-9.255,8.11$ \\
\hline Fear-Anger & -1.69173 .55() & 4.43 & -0.38 & 1832 & 0.70 & $-10.37,6.70$ \\
\hline Fear-Happy & $-5.64(169.60)$ & 4.43 & -1.27 & 1832 & 0.20 & $-14.32,3.04$ \\
\hline \multicolumn{7}{|c|}{ Expression effect - Manipulated Faces } \\
\hline Fear-manipulated & $-8.03(167.21)$ & 4.4 & -1.81 & 1832 & 0.07 & $-16.71,0.65$ \\
\hline Neutral-manipulated & $+15.04(182.61)$ & 6.26 & 2.46 & 1832 & $0.01^{*}$ & $3.13,27.68$ \\
\hline Anger-manipulated & $+13.28(180.49)$ & 6.26 & 2.12 & 1832 & $0.03^{*}$ & $1.00,25.56$ \\
\hline Happy-manipulated & $+10.90(178.11)$ & 6.26 & 1.74 & 1832 & 0.08 & $-1.37,23.18$ \\
\hline Main Effect of Manipulation & $(173.27-175.14)$ & 2.22 & 0.84 & 1838 & 0.40 & $-2.5,6.23$ \\
\hline \multicolumn{7}{|c|}{ (c) Saccadic responses to apparent-matched, broadband faces } \\
\hline \multicolumn{7}{|c|}{ Expression effect - Normal Faces } \\
\hline Intercept (Fear) & 172.22 & 5.14 & 33.05 & 1832 & $<.001$ & $162.14,182.30$ \\
\hline Fear-Neutral & $-0.52(171.70)$ & 4.40 & -0.12 & 1832 & 0.91 & $-9.14,8.11$ \\
\hline Fear-Anger & $-2.52(169.70)$ & 4.40 & -0.57 & 1832 & 0.57 & $-11.15,6.10$ \\
\hline Fear-Happy & $-0.59(171.63)$ & 4.40 & -0.13 & 1832 & 0.89 & $-9.22,8.03$ \\
\hline \multicolumn{7}{|c|}{ Expression effect - Manipulated Faces } \\
\hline Fear-manipulated & $2.72(174.94)$ & 4.40 & 0.62 & 1832 & 0.54 & $-5.90,11.34$ \\
\hline Neutral-manipulated & $-0.56(174.38)$ & 6.22 & -0.09 & 1832 & 0.93 & $-12.77,11.63$ \\
\hline Anger-manipulated & $3.80(178.18)$ & 6.22 & 0.61 & 1832 & 0.54 & $-8.40,16.00$ \\
\hline Happy-manipulated & $-3.67(171.26)$ & 6.22 & -0.59 & 1832 & 0.55 & $-15.88,8.52$ \\
\hline Main Effect of Manipulation & $(171.31-173.92)$ & 2.20 & 1.19 & 1838 & 0.24 & $-1.70,6.93$ \\
\hline
\end{tabular}

Table notes : ${ }^{*}$ indicates significant p-value. Sub-tables are for (a) raw, not-normalised, (b) RMS-normalised and (c) psychophysically normalised for apparent, perceived contrast. For each sub-table Fear is the intercept, and reports the estimated response time in milliseconds (ms) for the normal face condition. The face-format effect reflects the change in slope for the manipulated face, with fear as the reference and the estimated response time in (ms).

\section{Overall effects of contrast on faces' salience}

If it is the case that saccadic responses are determined by image salience, in the present study we should expect to see (i) fewer saccadic response time differences between 
Table 2. Linear mixed effects models (3) summarising saccadic responses for low-frequency facial stimuli, at each contrast condition.

\begin{tabular}{|l|l|l|l|l|l} 
Response estimate (ms) & SE & tStat & df & $p$ & CI (95\%)
\end{tabular}

(a) Saccadic responses to raw (unmatched), low-frequency faces

Expression effect - Normal Faces

\begin{tabular}{|l|l|l|l|l|l|l|}
\hline Intercept (Fear) & 177.66 & 5.97 & 29.78 & 1832 & $<.001$ & $165.96,189.36$ \\
\hline Fear-Neutral & $6.23(183.89)$ & 4.56 & 1.37 & 1832 & 0.17 & $-2.70,15.17$ \\
\hline Fear-Anger & $2.14(179.80)$ & 4.56 & 0.47 & 1832 & 0.64 & $-6.80,11.08$ \\
\hline Fear-Happy & $-0.36(177.30)$ & 4.56 & -0.08 & 1832 & 0.94 & $-9.30,8.58$ \\
\hline Expression effect - Manipulated Faces & $1.70(179.36)$ & 4.56 & 0.37 & 1832 & 0.71 & $-7.23,10.64$ \\
\hline Fear-manipulated & $-1.07(184.53)$ & 6.44 & -0.17 & 1832 & 0.87 & $-13.70,11.57$ \\
\hline Neutral-manipulated & $1.64(183.14)$ & 6.44 & 0.26 & 1832 & 0.80 & $-11.00,14.28$ \\
\hline Anger-manipulated & $1.00(180.00)$ & 6.44 & 0.15 & 1832 & 0.88 & $-11.64,13.63$ \\
\hline Happy-manipulated & 2.28 & 0.92. & 1838 & 0.36 & $-2.38,6.57$ \\
\hline Main Effect of Manipulation & $(179.66-181.76)$ &
\end{tabular}

(b) Saccadic responses to RMS-matched, low-frequency faces

\begin{tabular}{|l|l|l|l|l|l|l|}
\hline Expression effect - Normal Faces & 178.20 & 5.97 & 29.85 & 1832 & $<.001$ & $166.49,189.90$ \\
\hline Intercept (Fear) & $9.18(187.34)$ & 5.12 & 1.80 & 1832 & 0.07 & $-0.85,19.22$ \\
\hline Fear-Neutral & $-1.16(177.03)$ & 5.12 & -0.23 & 1832 & 0.82 & $-11.19,8.87$ \\
\hline Fear-Anger & $7.60(185.80)$ & 5.12 & 1.49 & 1832 & 0.14 & $-2.43,17.63$ \\
\hline Fear-Happy & $7.47(185.67)$ & 5.12 & 1.46 & 1832 & 0.14 & $-2.56,17.50$ \\
\hline Expression effect - Manipulated Faces & 7.23 & -0.94 & 1832 & 0.35 & $-21.00,7.38$ \\
\hline Fear-manipulated & $-6.81(188.04)$ & 7.23 & 0.27 & 1832 & 0.79 & $-12.27,16.11$ \\
\hline Neutral-manipulated & $1.92(186.43)$ & 7.23 & -1.76 & 1832 & 0.08 & $-26.95,1.43$ \\
\hline Anger-manipulated & $-12.76(180.50)$ & 2.56 & 1.19 & 1838 & 0.23 & $-1.97,8.09$ \\
\hline Happy-manipulated & $(182.10-185.16)$ & \multicolumn{5}{l|}{} \\
\hline Main Effect of Manipulation &
\end{tabular}

(c) Saccadic responses to apparent-matched, low-frequency faces

Expression effect - Normal Faces

\begin{tabular}{|l|l|l|l|l|l|l|}
\hline Intercept (Fear) & 178.41 & 5.47 & 32.64 & 1832 & $<.001$ & $167.69,189.13$ \\
\hline Fear-Neutral & $-6.19(172.22)$ & 4.42 & -1.40 & 1832 & 0.16 & $-14.85,2.47$ \\
\hline Fear-Anger & $3.71(182.12)$ & 4.42 & 0.84 & 1832 & 0.40 & $-4.95,12.37$ \\
\hline Fear-Happy & $-1.15(177.26)$ & 4.42 & -0.26 & 1832 & 0.79 & $-9.81,7.51$ \\
\hline Expression effect - Manipulated Faces & $4.79(183.20)$ & 4.42 & 1.08 & 1832 & 0.28 & $-3.87,13.45$ \\
\hline Fear-manipulated & $10.85(187.86)$ & 6.25 & 1.74 & 1832 & 0.08 & $-1.40,23.10$ \\
\hline Neutral-manipulated & $0.38(187.29)$ & 6.25 & 0.06 & 1832 & 0.95 & $-11.87,12.63$ \\
\hline Anger-manipulated & $3.73(185.79)$ & 6.25 & 0.60 & 1832 & 0.55 & $-8.52,15.99$ \\
\hline Happy-manipulated & 2.21 & 3.86 & 1838 & $<.001^{*}$ & $4.19,12.87$ \\
\hline Main Effect of Manipulation & $(177.5-186.03)$ &
\end{tabular}

Table notes : ${ }^{*}$ indicates significant p-value. Sub-tables are (a) raw, not-normalised, (b) RMS-normalised, (c) psychophysically normalised for apparent, perceived contrast. For each sub-table Fear is the intercept, and reports the response time in milliseconds (ms). 

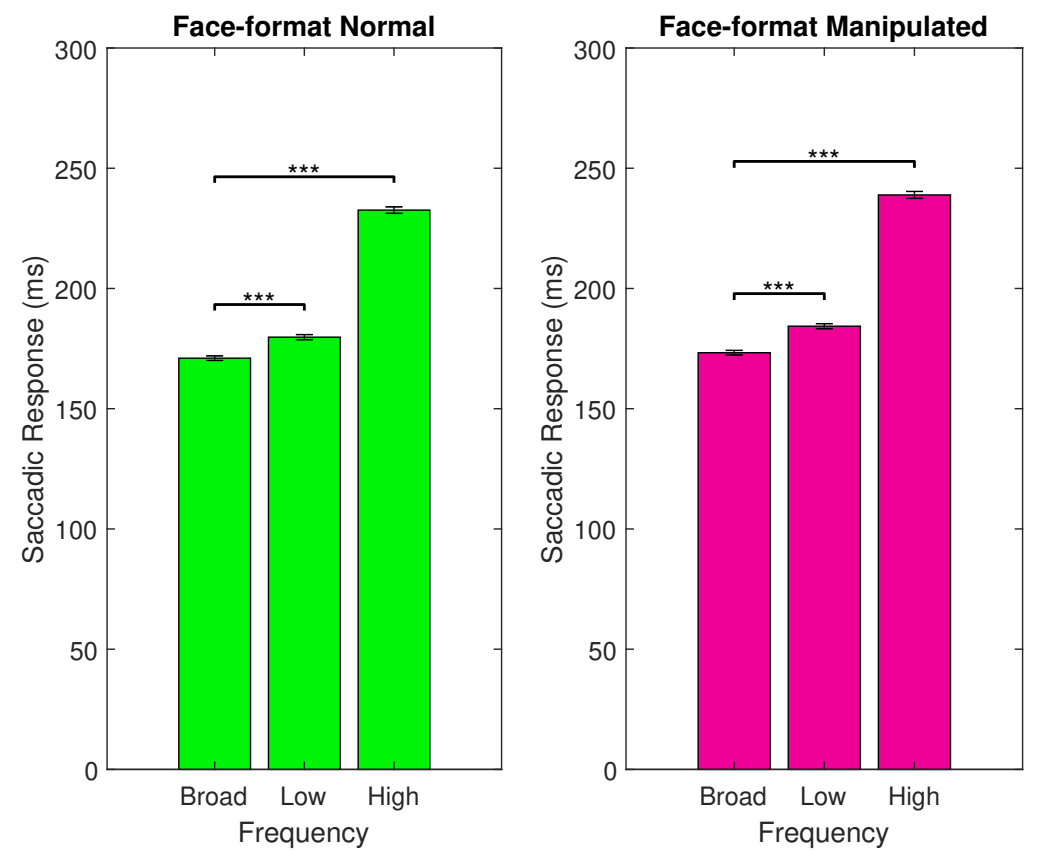

Fig 6. Saccadic responses (ms) for Normal and Manipulated faces by spatial frequency (collapsed across emotion and contrast). Error bars are 1士 SEM.

expressions matched for apparent contrast -note that this finding was confirmed above, 307 (ii) slower saccadic response times for high-frequency-filtered stimuli overall, because contrast reduces with increasing frequency, (iii) and faster saccadic responses overall for raw, non-normalised facial stimuli, because this stimulus set were overall higher in contrast compared to faces normalised for RMS or apparent, perceived contrast. To address these hypotheses a linear mixed effects model was conducted independently for each frequency and face-format using the 'fitlme' function in MATLAB, where 'participant' was the random effect, and contrast was the fixed effect.

The overall effect of faces' spatial frequency on saccadic responses is summarised in Table 3, and depicted in Fig 6. For normally presented, upright faces, saccadic response times are slower for high-frequency-filtered versions of faces, and fastest for intact, broadband versions of faces. The same effects are observed for control faces, when presented in manipulated format- an expected effect given that both normal and control faces share the same contrast and spatial frequency content. In terms of contrast normalisation-related effects, for broadband stimuli - collapsed across expression- , faces that were not normalised for contrast received significantly faster saccadic response times compared to RMS normalised stimuli, and while the same tended to be true when compared to stimuli normalised for apparent contrast, this difference did not reach statistical significance. Saccadic response times to low-frequency-filtered stimuli did not significantly differ according to the way in which they were normalised for contrast, and for high-frequency-filtered stimuli (noting again that data are missing for RMS-normalised condition), raw high frequency stimuli again received faster saccadic responses compared to the same stimuli when normalised for apparent contrast. The same model was conducted for stimuli when presented in manipulated format (inverted, reversed luminance polarity), and here only raw high frequency stimuli received overall faster responses compared to those normalised for apparent contrast. These findings are summarised in Table 4 , and displayed in Fig 7. 
Table 3.

\begin{tabular}{|c|c|c|c|c|c|c|}
\hline & Response estimate (ms) & SE & tStat & df & $p$ & CI (95\%) \\
\hline \multicolumn{7}{|c|}{ (a) Saccadic responses to normal faces } \\
\hline Broad & 171.02 & 4.10 & 41.68 & 8277 & $<.001$ & $162.98,179.06$ \\
\hline Low & +8.73 & 1.53 & 5.72 & 8277 & $<.001^{*}$ & $5.74,11.73$ \\
\hline High & +61.57 & 1.53 & 40.32 & 8277 & $<.001^{*}$ & $58.58,64.56$ \\
\hline \multicolumn{7}{|c|}{ (b) Saccadic responses to manipulated faces } \\
\hline Broad & 173.28 & 4.21 & 41.21 & 8277 & $<.001$ & $165.04,181.52$ \\
\hline Low & +11.04 & 1.57 & 7.03 & 8277 & $<.001^{*}$ & $7.96,14.12$ \\
\hline High & +65.62 & 1.57 & 41.78 & 8277 & $<.001^{*}$ & $62.54,68.70$ \\
\hline
\end{tabular}

Table notes : * indicates significant p-value. Sub-tables are (a) saccadic responses to normal faces at each frequency condition, and (b) saccadic responses to manipulated faces at each frequency condition. For each table the intact, broadband condition is the intercept, and reports the response time in milliseconds $(\mathrm{ms})$.

\section{Summary of findings}

Findings show that overall, saccadic initiation is faster for facial stimuli with higher contrast, regardless of its emotional content. This is evidenced by faster saccadic response times for broadband (versus low and high frequency) stimuli, and raw stimuli that were not normalised for contrast (versus RMS and apparent matched stimuli); both of which contain the highest contrast of all frequency and contrast conditions. Moreover, saccadic responses are overall slower for high-frequency-filtered stimuli, regardless of expression and contrast normalisation, further showing that when physical contrast is lost with increasing frequency, saccades are slower to be deployed. In terms of biased saccadic responses for fearful facial expressions, no effects were observed for typical, upright stimuli, regardless of their spatial frequency content. In some instances, however, we observed mixed findings for fearful faces presented in manipulated format. For example, we observed a saccade bias for manipulated broadband fearful faces when normalised for RMS contrast that becomes a disadvantage when not normalised for contrast at all. Importantly, though we observed few differences in saccadic responses to fear compared to other faces, these differences almost completely vanished across all frequency conditions when expressions were normalised for their apparent, perceived contrast. Finally, there was little evidence to suggest that normally-presented faces received faster saccadic response times compared to manipulated counterparts, with the exception that saccadic response times were overall faster for low frequency normal faces when normalised for apparent contrast, and high frequency normal faces when not normalised at all. These implication of these findings for the threat bias literature, and for image standardisation procedures, are discussed in the following section.

\section{Discussion}

The present study was motivated by the threat bias literature, particularly that which proposes a unique bias for responding to, and processing, fearful facial stimuli. Findings thus far are relatively mixed, and are derived from a range of experimental paradigms $2,3,7,9,11,14,16,19,23$. From these findings, the role of spatial frequency, face presentation duration, and response mode are unclear. Saccadic latency is considered to be a favourable approach over manual response modes, because it indexes implicit and reflexive responses to stimuli as they become available in the visual field [6, 20]. Despite this, the role of low-level image information on saccadic responses 
Table 4.

\begin{tabular}{|c|c|c|c|c|c|c|}
\hline & Response estimate (ms) & SE & tStat & df & $p$ & CI $(95 \%)$ \\
\hline \multicolumn{7}{|c|}{ (a) Saccadic responses to normal faces } \\
\hline \multicolumn{7}{|c|}{ Broadband } \\
\hline Raw & 168.48 & 4.49 & 37.51 & 2757 & $<.001$ & $159.67,177.28$ \\
\hline Rms & 4.79 & 2.15 & 2.23 & 2757 & $0.026^{*}$ & $0.57,9.00$ \\
\hline Apparent & 2.84 & 2.15 & 1.32 & 2757 & 0.187 & $-1.38,7.05$ \\
\hline \multicolumn{7}{|c|}{ Low Frequency } \\
\hline Raw & 179.66 & 5.11 & 35.16 & 2757 & $<.001$ & $169.64,189.68$ \\
\hline Rms & 2.44 & 2.37 & 1.03 & 2757 & 0.304 & $-2.21,7.09$ \\
\hline Apparent & -2.16 & 2.37 & -0.91 & 2757 & 0.363 & $-6.81,2.49$ \\
\hline \multicolumn{7}{|c|}{ High Frequency } \\
\hline Raw & 217.97 & 4.92 & 44.30 & 2757 & $<.001$ & $208.32,227.62$ \\
\hline Rms & 20.49 & 3.13 & 6.54 & 2757 & $<.001^{*}$ & $14.34,26.63$ \\
\hline Apparent & 23.37 & 3.13 & 7.46 & 2757 & $<.001^{*}$ & $17.23,29.52$ \\
\hline
\end{tabular}

\begin{tabular}{|c|c|c|c|c|c|c|}
\hline \multicolumn{7}{|c|}{ (b) Saccadic responses to manipulated faces } \\
\hline \multicolumn{7}{|c|}{ Broadband } \\
\hline Raw & 170.78 & 4.52 & 37.75 & 2757 & $<.001$ & $161.91,179.65$ \\
\hline Rms & 4.35 & 2.17 & 2.01 & 2757 & $0.045^{*}$ & $0.10,8.60$ \\
\hline Apparent & 3.14 & 2.17 & 1.45 & 2757 & 0.147 & $-1.11,7.39$ \\
\hline \multicolumn{7}{|c|}{ Low Frequency } \\
\hline Raw & 181.76 & 4.85 & 37.45 & 2757 & $<.001$ & $172.24,191.27$ \\
\hline Rms & 3.40 & 2.34 & 1.45 & 2757 & 0.147 & $-1.19,8.00$ \\
\hline Apparent & 4.28 & 2.34 & 1.82 & 2757 & 0.068 & $-0.32,8.87$ \\
\hline \multicolumn{7}{|c|}{ High Frequency } \\
\hline Raw & 225.87 & 5.22 & 43.30 & 2757 & $<.001$ & $215.64,236.10$ \\
\hline Apparent & 17.57 & 3.35 & 5.24 & 2757 & $<.001^{*}$ & $10.99,24.15$ \\
\hline
\end{tabular}

Table notes : ${ }^{*}$ indicates significant p-value. Sub-tables are (a) saccadic responses to normal faces at each frequency condition, and within this, when presented in raw (not normalised) format, RMS normalised format, or apparent-perceived format, and (b) the same results for manipulated versions of faces. For each table the raw (not normalised) frequency version of the face is the intercept, and reports the response time in milliseconds $(\mathrm{ms})$. 
Table 5. Linear mixed effects models (3) summarising saccadic responses for high-frequency facial stimuli, at each contrast condition.

\begin{tabular}{|l|l|l|l|l|l|l|}
\hline & Response estimate (ms) & SE & tStat & df & $p$ & CI (95\%) \\
\hline \multicolumn{7}{|l|}{} \\
(a) Saccadic responses to raw (unmatched), high-frequency faces \\
\hline Expression effect - Normal Faces \\
\hline Intercept (Fear) & 217.27 & 6.40 & 33.97 & 1832 & $<.001$ & $204.73,229.82$ \\
\hline Fear-Neutral & $4.07(221.34)$ & 6.01 & 0.68 & 1832 & 0.50 & $-7.71,15.86$ \\
\hline Fear-Anger & $1.99(219.26)$ & 6.01 & 0.33 & 1832 & 0.74 & $-9.80,13.77$ \\
\hline Fear-Happy & $-3.28(213.99)$ & 6.01 & -0.55 & 1832 & 0.59 & $-15.06,8.50$ \\
\hline Expression effect - Manipulaten Faces & $6.47(223.74)$ & 6.01 & 1.08 & 1832 & 0.28 & $-5.31,18.26$ \\
\hline Fear-manipulated & $-3.11(223.74)$ & 8.50 & -0.37 & 1832 & 0.71 & $-19.77,13.55$ \\
\hline Neutral-manipulated & $1.87(225.61)$ & 8.50 & 0.22 & 1832 & 0.83 & $-14.80,18.53$ \\
\hline Anger-manipulated & $6.95(230.69)$ & 8.50 & 0.82 & 1832 & 0.41 & $-9.71,23.61$ \\
\hline Happy-manipulated & $(217.97-225.87)$ & 3.01 & 2.63 & 1838 & $0.008^{*}$ & $2.01,13.80$ \\
\hline Main Effect of Manipulation &
\end{tabular}

(b) Saccadic responses to RMS-matched, high-frequency faces

Corrupted Dataset

(c) Saccadic responses to apparent-matched, high-frequency faces

Expression effect - Normal Faces

\begin{tabular}{|l|l|l|l|l|l|l|}
\hline Intercept (Fear) & 245.10 & 6.74 & 36.37 & 1832 & $<.001$ & $231.88,258.32$ \\
\hline Fear-Neutral & $-5.45(249.17)$ & 6.81 & -0.80 & 1832 & 0.42 & $-18.80,7.90$ \\
\hline Fear-Anger & $-3.16(247.09)$ & 6.81 & -0.46 & 1832 & 0.64 & $-16.51,10.19$ \\
\hline Fear-Happy & $-6.42(241.82)$ & 6.81 & -0.94 & 1832 & 0.35 & $-19.77,6.93$ \\
\hline Expression effect - Manipulated Faces & $-0.52(251.57)$ & 6.81 & -0.08 & 1832 & 0.94 & $-13.87,12.83$ \\
\hline Fear-manipulated & $6.38(248.47)$ & 9.63 & 0.66 & 1832 & 0.51 & $-12.50,25.26$ \\
\hline Neutral-manipulated & $5.67(253.44)$ & 9.63 & 0.59 & 1832 & 0.56 & $-13.21,24.55$ \\
\hline Anger-manipulated & $-1.57(258.52)$ & 9.63 & -0.16 & 1832 & 0.87 & $-20.45,17.31$ \\
\hline Happy-manipulated & $(241.34-243.44)$ & 3.41 & 0.62 & 1838 & 0.53 & $-4.59,8.78$ \\
\hline Main Effect of Manipulation &
\end{tabular}

Table notes : ${ }^{*}$ indicates significant p-value. Sub-tables are (a) raw, not-normalised, (b) RMS-normalised, (c) psychophysically normalised for apparent, perceived contrast. For each sub-table Fear is the intercept, and reports the response time in milliseconds (ms). Note the absent results for condition (b) Saccadic Responses to RMS-matched, high frequency faces. 

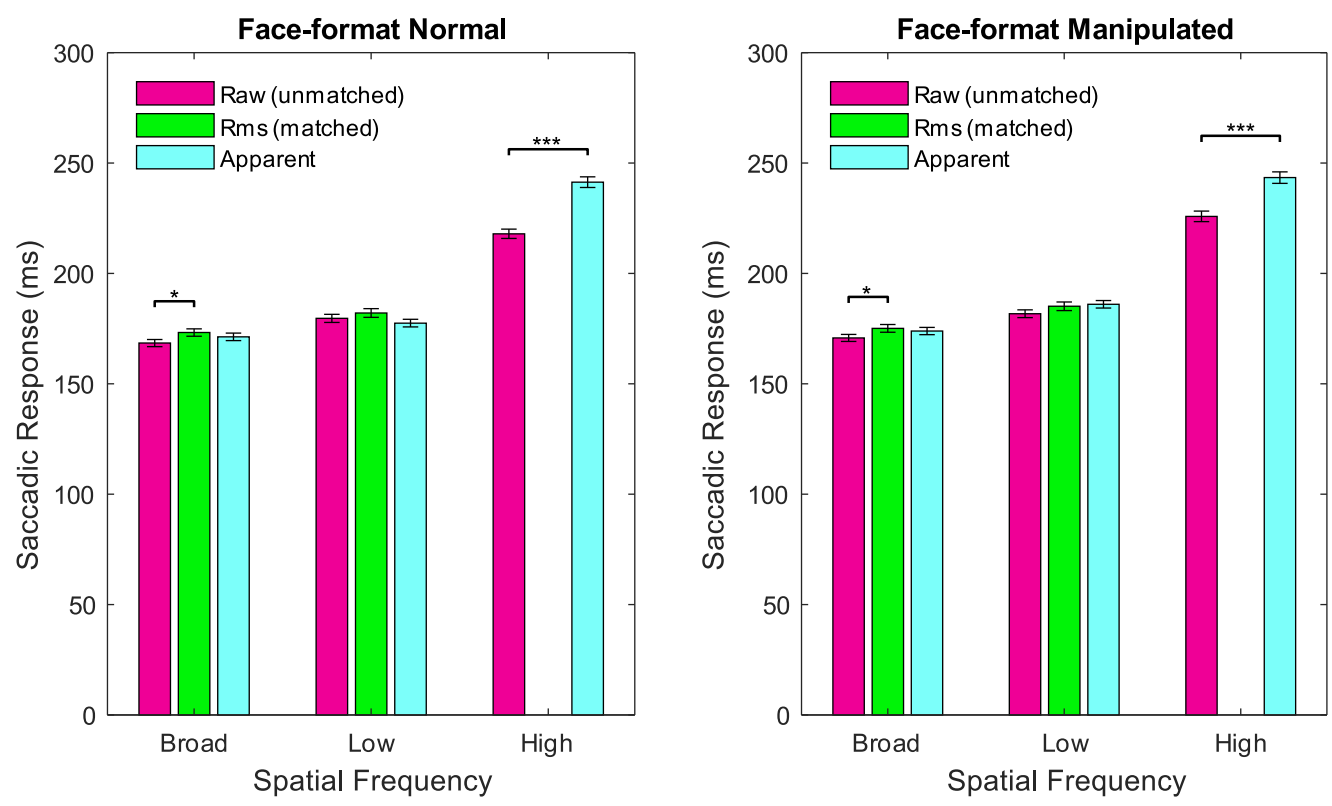

Fig 7. Saccadic responses (ms) for Normal and Manipulated faces (collapsed across

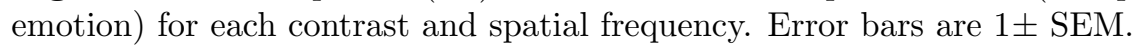

to facial emotional displays, including spatial frequency and contrast, remains relatively unexplored. To investigate this, we measured saccadic response times for neutral, angry, fearful and happy faces, when presented in broadband format, or filtered to contain low versus high frequency information. To assess the role of contrast including normalised contrast, perceived contrast, and naturally-occurring differences in physical contrast between expressions, faces were shown in their raw (not normalised) format, when normalised for RMS contrast, and when normalised for apparent, perceived contrast. Findings from the present study can be separated into three main areas: (i) findings regarding the threat bias for spatially filtered fear expressions, (ii) the overall effect of contrast on saccadic responses, and (iii) detectability of upright faces versus those subjected to spatial inversion and luminance polarity reversal.

\section{No saccadic biases for spatially-filtered fear expressions}

For typical, broadband facial stimuli, saccadic response times did not differ for fearful facial displays compared to neutral, angry, or happy faces. Although this finding (or lack thereof) is at odds with studies showing faster detection and processing time associated with fearful faces $3,8,13,16,18,22$, it contributes to, and is consistent with, a growing body of literature showing the absence of a threat bias for fearful facial displays $8,10,14,19,31,32$. We also did not find any evidence of detectability advantages for fearful expressions filtered to contain low spatial frequency information, and in fact saccadic response times for fearful faces did not differ compared to counterpart faces in either low or high frequency conditions. There is some disagreement regarding the role of spatial frequency for rapid fear processing, where Bannerman et al. 13] show response biases for low-frequency-filtered fearful faces, and Stein et al. [18] show biases only for high-frequency-filtered versions of faces, the present study does not support either body of evidence. It is, however, necessary to note here that in a small number of cases, we observed both faster and slower saccadic response 
times for control fearful faces (e.g. when spatially inverted and subjected to reversed luminance polarity). The fact that these effects differed according to spatial frequency content and contrast condition suggests a role of low-level image information in determining these effects, as opposed to the face as a fearful expression. Together, findings from the present study suggest that fearful expressions do not receive prioritised access to attentional resources, and instead show that saccadic initiation is influenced not by the emotional content of faces, or their expression-related low-level differences, but by overall salience of the face as an image.

\section{Saccadic responses are influenced by global image contrast}

To reiterate, we know two important things about the faces used in this study, (i) faces in the 'raw' condition contained naturally-occurring and expression-related differences in RMS contrast, where fearful faces, already naturally lower in contrast compared to other expressions, became disproportionately lower in contrast as their spatial frequency increased [19], and (ii) fearful faces in the 'apparent matched' condition were subjected to lower RMS contrast settings compared to other faces, such that fearful and counterpart faces contained varying contrasts required to appear perceptually the same, and these differences too become enhanced as faces' spatial frequency content increased [26] -in other words, fearful faces consistently require less than other expressions to appear perceptually the same as a reference stimulus, and the magnitude of this effect becomes more pronounced as their spatial frequency content is increased. On the other hand, expressions in the raw face condition naturally differ in terms of their RMS contrast, and were overall higher in physical contrast compared to both those normalised for apparent contrast, and those normalised for RMS contrast. This information allows us to measure the degree to which saccadic responses were influenced by the overall physical composition of faces, independently of expression-related effects. Here, we show that response times for detecting raw stimuli, containing the highest average contrast, were generally faster than for faces containing lower contrast, suggesting that saccades are 'drawn to' physically salient facial images, regardless of their emotional relevance. This 'image effect' is further supported by the finding that saccadic response times were also slowest for face images composed of high frequency information, because this stimulus set was disadvantaged by contrast losses through spatial filtering. Finally, previous literature regarding the extent to which low frequency-dependent fear bias effects are accounted for by contrast sensitivity effects (greater contrast at spatial frequency ranges in line with 'low frequency' conditions), are also not supported here. This is because the present findings show that saccadic responses are overall faster for broadband versus low frequency facial stimuli.

It is difficult to directly compare the contrast-related effects found in the present study to those from similar experimental designs. Details regarding contrast differences between images, the extent to which they are normalised, and for which contrast metrics, are not always consistently reported in the literature. However, the role of contrast alone can not account for variable findings. Findings from Bannerman et al. 13, for example, show clear saccadic biases for fearful faces that were not normalised for contrast. Given this, we should have expected to observe the same effect in the present broadband condition, but we did not. Moreover, Khalid et al. 11] did normalise facial stimuli for contrast, finding no fear bias compared to neutral faces, and Webb and Hibbard [23] found that contrast normalisation of facial stimuli facilitates biases for detecting fear expressions. Notably, these examples come from studies using different experimental parameters, and it is likely that the role of image contrast differs relative to the task at hand. Recently, there has been an emerging view that contrast normalisation can inadvertently both boost 23,26 and impede 27 performance on 
facial emotion tasks. While the present findings do not show a facilitatory effect of contrast normalisation on fear detection biases [19,23,26], they do suggest that improved performance for fearful faces not normalised for contrast 27] may be due to the fact that these faces, compared to those normalised for physical or apparent contrast, are already advantaged in terms of containing higher contrast.

\section{Saccades do not always rely on holistic facial information}

A final area of interest was the role of face format, or the use of control facial stimuli, on saccadic response times. The present study created control versions of facial stimuli using spatial inversion combined with luminance polarity reversal, a combined treatment known to significantly impede facial recognition [22]. Recently, however, some evidence suggests that luminance polarity reversal may, in some cases, inadvertently increase the perceived salience of a control face (for review, see 29]. Saccadic latency is therefore an ideal candidate for exploring these effects, particularly because findings regarding the effects of holistic information in upright versus inverted faces for saccadic responses are fairly mixed. Khalid et al. [11], for example, found that performance was better for inverted over upright facial stimuli, while Bannerman et al. [12 showed the opposite. In the present study, saccadic response times did not differ according to the format of typical, broadband facial stimuli, such that the combination of spatial inversion and luminance polarity reversal did not influence their detectability. This finding is surprising, because it suggests that saccadic latency is indifferent to the holistic information present in upright faces; an unexpected outcome given the renowned face inversion effect that is consistently observed for regular, unfiltered faces 29,33. Upright (luminance retained) faces did, however, receive faster saccadic response times when faces were low- and high-frequency-filtered, but this was influenced by the use of contrast normalisation, where detectability advantages for upright low frequency faces relied on their consistency in terms of apparent, perceived contrast, while detectability advances for high frequency faces relied on naturally-occurring contrast differences between expressions. These findings are consistent with previous literature, in that they demonstrate an unclear interaction between contrast, luminance, and orientation for facial emotion processing [23,26], and contribute to recent literature regarding the role of luminance polarity reversal, showing that reflexive eye movements are not facilitated by an increase of local dark regions 29,34

\section{Future replication}

A replication of the present study might investigate in more detail the role of image presentation duration, particularly because the degree to which threat biases occur in response to transient versus consciously-evaluated faces remains unclear. Bannerman and colleagues 12, 13] suggest that for longer face durations (500 ms and above), manual rather than saccadic responses are the preferred response mode, and that saccadic responses are more appropriately explored using very short stimulus presentations, below $200 \mathrm{~ms}$. Despite this, Seitz and colleagues [21], for example, showed that the eyes of fearful faces were located faster compared to happy faces when available for $150 \mathrm{~ms}$, but not compared to neutral or angry faces, showing that biases for detecting threat-relevant information in fear expressions is possible in a simple localisation task, even at $150 \mathrm{~ms}$ durations. Similarly, fearful face precues shown for both 100 and $500 \mathrm{~ms}$ have also been shown to have no effect on search efficiency, 31, while other instances evidence improved search efficiency following exposure to fearful faces for 500 as opposed to $100 \mathrm{~ms}$ 32]. Moreover, regardless of the stimulus duration length, it is generally accepted that longer durations (500 and $5000 \mathrm{~ms}$ ) are capable of eliciting overall faster saccadic responses for stimuli (regardless of expression) compared to manual ones 12,21, suggesting that this mode of response can generally sample, and 


\section{Conclusion}

Findings show that saccadic eye movements are largely indifferent to the emotional content of facial stimuli, and that factors including image contrast and spatial frequency are more likely to influence the speed at which saccades are deployed. These findings contribute to a growing body of literature demonstrating an absence of threat bias effects for fearful faces, and further demonstrate that the statistical image properties of facial stimuli, and the degree to which they are adjusted during image standardisation, can have significant effects on behavioural performance on facial emotion tasks.

\section{Supporting information}

\section{Acknowledgments}

\section{References}

1. Öhman A, Mineka S. Fears, phobias, and preparedness: toward an evolved module of fear and fear learning. Psychological review. 2001;108(3):483.

2. Vuilleumier P, Armony JL, Driver J, Dolan RJ. Distinct spatial frequency sensitivities for processing faces and emotional expressions. Nature neuroscience. 2003;6(6):624-631.

3. Carlson JM, Reinke KS. Masked fearful faces modulate the orienting of covert spatial attention. Emotion. 2008;8(4):522.

4. Susskind JM, Lee DH, Cusi A, Feiman R, Grabski W, Anderson AK. Expressing fear enhances sensory acquisition. Nature neuroscience. 2008;11(7):843.

5. Pourtois G, Grandjean D, Sander D, Vuilleumier P. Electrophysiological correlates of rapid spatial orienting towards fearful faces. Cerebral cortex. 2004;14(6):619-633.

6. Bannerman RL, Milders M, Sahraie A. Attentional bias to brief threat-related faces revealed by saccadic eye movements. Emotion. 2010;10(5):733.

7. Pourtois G, Schwartz S, Seghier ML, Lazeyras F, Vuilleumier P. Neural systems for orienting attention to the location of threat signals: an event-related fMRI study. Neuroimage. 2006;31(2):920-933.

8. Holmes A, Green S, Vuilleumier P. The involvement of distinct visual channels in rapid attention towards fearful facial expressions. Cognition \& Emotion. 2005;19(6):899-922.

9. Fox E, Russo R, Dutton K. Attentional bias for threat: Evidence for delayed disengagement from emotional faces. Cognition \& emotion. 2002;16(3):355-379.

10. Mogg K, Garner M, Bradley BP. Anxiety and orienting of gaze to angry and fearful faces. Biological psychology. 2007;76(3):163-169. 
11. Khalid S, Horstmann G, Ditye T, Ansorge U. Measuring the emotion-specificity of rapid stimulus-driven attraction of attention to fearful faces: evidence from emotion categorization and a comparison with disgusted faces. Psychological research. 2017;81(2):508-523.

12. Bannerman RL, Milders M, De Gelder B, Sahraie A. Orienting to threat: faster localization of fearful facial expressions and body postures revealed by saccadic eye movements. Proceedings of the Royal Society B: Biological Sciences. 2009;276(1662):1635-1641.

13. Bannerman RL, Hibbard PB, Chalmers K, Sahraie A. Saccadic latency is modulated by emotional content of spatially filtered face stimuli. Emotion. 2012;12(6):1384.

14. Bayle DJ, Schoendorff B, Hénaff MA, Krolak-Salmon P. Emotional facial expression detection in the peripheral visual field. PloS one. 2011;6(6):e21584.

15. Becker MW, Detweiler-Bedell B. Short article: Early detection and avoidance of threatening faces during passive viewing. Quarterly Journal of Experimental Psychology. 2009;62(7):1257-1264.

16. Yang E, Zald DH, Blake R. Fearful expressions gain preferential access to awareness during continuous flash suppression. Emotion. 2007;7(4):882.

17. Hedger N, Adams WJ, Garner M. Fearful faces have a sensory advantage in the competition for awareness. Journal of Experimental Psychology: Human Perception and Performance. 2015;41(6):1748.

18. Stein T, Seymour K, Hebart MN, Sterzer P. Rapid fear detection relies on high spatial frequencies. Psychological science. 2014;25(2):566-574.

19. Webb AL, Hibbard PB. The effect of facial expression on contrast sensitivity: A behavioural investigation and extension of Hedger, Adams \& Garner (2015). PloS one. 2019;14(11):e0205621.

20. Reuter-Lorenz PA, Hughes HC, Fendrich R. The reduction of saccadic latency by prior offset of the fixation point: an analysis of the gap effect. Perception \& psychophysics. 1991;49(2):167-175.

21. Seitz KI, Leitenstorfer J, Krauch M, Hillmann K, Boll S, Ueltzhoeffer K, et al. An eye-tracking study of interpersonal threat sensitivity and adverse childhood experiences in borderline personality disorder. Borderline personality disorder and emotion dysregulation. 2021;8(1):1-12.

22. Gray KL, Adams WJ, Hedger N, Newton KE, Garner M. Faces and awareness: low-level, not emotional factors determine perceptual dominance. Emotion. 2013;13(3):537.

23. Webb AL, Hibbard PB. Suppression durations for facial expressions under breaking continuous flash suppression: effects of faces' low-level image properties. Scientific reports. 2020;10(1):1-11.

24. Stuit S, Kootstra T, Terburg D, van den Boomen C, van der Smagt M, Kenemans J, et al. The image features of emotional faces that predict the initial eye movement to a face. Scientific Reports. 2021;11(1):1-14. 
25. Lundqvist D, Flykt A, Öhman A. The Karolinska directed emotional faces (KDEF). CD ROM from Department of Clinical Neuroscience, Psychology section, Karolinska Institutet. 1998;91(630):2-2.

26. Webb AL, Hibbard PB, O'Gorman R. Contrast normalisation masks natural expression-related differences and artificially enhances the perceived salience of fear expressions. PloS one. 2020;15(6):e0234513.

27. Menzel C, Redies C, Hayn-Leichsenring GU. Low-level image properties in facial expressions. Acta psychologica. 2018;188:74-83.

28. Vlamings PH, Goffaux V, Kemner C. Is the early modulation of brain activity by fearful facial expressions primarily mediated by coarse low spatial frequency information? Journal of vision. 2009;9(5):12-12.

29. Webb AL. Reversing the Luminance Polarity of Control Faces: Why Are Some Negative Faces Harder to Recognize, but Easier to See? Frontiers in Psychology. 2021;11:3901.

30. Brainard D. Psychophysics software for use with MATLAB. Spatial Vision. 1997;10(4):433-436.

31. Kunar MA, Watson DG, Cole L, Cox A. Negative emotional stimuli reduce contextual cueing but not response times in inefficient search. Quarterly Journal of Experimental Psychology. 2014;67(2):377-393.

32. Olatunji BO, Ciesielski BG, Armstrong T, Zald DH. Emotional expressions and visual search efficiency: Specificity and effects of anxiety symptoms. Emotion. 2011;11(5):1073.

33. Farah MJ, Tanaka JW, Drain HM. What causes the face inversion effect? Journal of Experimental Psychology: Human perception and performance. 1995;21(3):628.

34. Haun AM, Peli E. Perceived contrast in complex images. Journal of vision. 2013;13(13):3-3. 